\title{
基于交变磁场红外热像的磁性水凝胶缺陷检测
}

\author{
乔严程 ${ }^{1,2}$ 解社娟 ${ }^{1,2}$ 全宗飞 ${ }^{1,2}$ 唐敬达 ${ }^{1}$ 许盼盼 $^{1,2}$ \\ 李 骥 ${ }^{3}$ 李丽娟 ${ }^{3}$ 陈振茂 ${ }^{1,2}$
}

(1. 西安交通大学机械结构强度与振动国家重点实验室 西安 710049;

2. 西安交通大学陕西省无损检测结构完整性评价工程技术中心 西安 710049;

3. 中国核动力研究设计院 成都 610213)

摘要：磁性水凝胶是具有磁性纳米粒子的水凝胶, 具有独特的磁响应特性, 被广泛应用于组织工程支架、生物工程细胞分离、 药物载体和环境工程等方面。磁性水凝胶的磁性纳米粒子的分布对其磁响应有着决定性的作用, 因此, 对于磁性水凝胶的磁 性均匀性分析是十分必要的。目前, 对于磁性水凝胶的无损检测手段基本空白, 鲜见文献报道。磁性水凝胶中磁性纳米粒子 在交变磁场作用下由于弛豫作用会产生热量。基于以上研究背景, 研究针对磁性水凝胶材料开发一种新型无损检测手段：基 于交变磁场的红外热成像检测方法。通过对磁性水凝胶和铜粉硅胶板的检测, 验证磁性水凝胶在交变磁场作用下的发热原理; 通过对不同浓度磁性水凝胶的检测, 验证利用交变磁场的红外热成像对磁性水凝胶中磁性纳米粒子均匀性分析的可行性, 并 对磁性水凝胶中磁性粒子分布不均、气泡缺陷分别进行检测, 结果显示均能较好地检出; 基于理论分析得出磁性水凝胶在交 变磁场作用下的表面温升速率与其磁性纳米粒子浓度存在线性关系的猜想，通过对不同浓度水凝胶的检测得到的数据进行拟 合，证实磁性水凝胶中磁性纳米粒子的浓度与其表面温升速率存在线性关系。

关键词: 磁性水凝胶; 磁性纳米粒子; 缺陷检测; 交变磁场; 红外热成像

中图分类号: $\mathrm{TB} 34$

\section{Magnetic Hydrogel Defect Detection Based on Infrared Thermal Image of Alternating Magnetic Field}

\author{
QIAO Yancheng $^{1,2}$ XIE Shejuan ${ }^{1,2}$ TONG Zongfei ${ }^{1,2}$ TANG Jingda ${ }^{1} \quad$ XU Panpan $^{1,2}$ \\ $\mathrm{LI} \mathrm{Ji}^{3} \quad$ LI Lijuan ${ }^{3}$ CHEN Zhenmao ${ }^{1,2}$
}

(1. Xi'an Jiaotong University, State Key Laboratory for Strength and Vibration of Mechanical Structures, Xi'an 710049;

2. Xi'an Jiaotong University, Shaanxi Engineering Research Center of Nondestructive

Testing and Structural Integrity Evaluation, Xi'an 710049;

3. Nuclear Power Institute of China, Chengdu 610213)

\begin{abstract}
Magnetic hydrogel, a hydrogel with magnetic nanoparticles and unique magnetic response characteristics, has been widely used in fields of tissue engineering scaffolds, bioengineered cell separation, drug carriers, and environmental engineering. The distribution of magnetic nanoparticles in magnetic hydrogels plays a decisive role in their magnetic responses, which makes the analysis of the magnetic homogeneity of magnetic hydrogels a necessity. Yet there still lacks sufficient research or practice in non-destructive testing of magnetic hydrogels and has not been reported in the literature. Based on the backgrounds above, a novel non-destructive testing method for magnetic hydrogel defect detection has been developed, that is, the infrared thermal imaging method based on alternating magnetic field. The specific work is as follows. The magnetic hydrogel and copper powder silica gel plate have been detected, and furthermore, the feasibility of the principle of the magnetic hydrogel's getting heated has been verified under the alternating magnetic field. through the detection of magnetic hydrogels with different concentrations, the feasibility of using alternating magnetic fields infrared for The uniformity analysis of magnetic nanoparticles in magnetic hydrogels is verified. The uneven distribution of magnetic particles and bubble defect have also been detected and it has been found that the method works in the above detections. Based on theoretical analysis, it is deduced that there could be a linear relationship between the surface temperature rise rate of the magnetic hydrogel and the concentration
\end{abstract}

\footnotetext{
* 国家自然科学基金(51877163, 11927801)、反应堆燃料及材料重点实验室基金、 新疆维吾尔自治区自然科学基金(2019D01A76)、中国博士后科学基金 (2018M641035)、陕西省高校科协青年人才托举计划(20170601)和远东无损检 测新技术论坛创新人才支持计划资助项目。20191001 收到初稿, 20200331 收 到修改稿
} 
of the magnetic nanoparticles under the alternating magnetic field. Through fitting the measured data of different concentration hydrogel, the above deduction is further proved.

Key words: magnetic hydrogels; magnetic nanoparticle; defect detection; alternating magnetic field; infrared thermal imaging

\section{0 前言}

磁性水凝胶是具有磁性纳米粒子可对外加磁场 做出响应的一种水凝胶, 在软机器人 ${ }^{[1]}$ 、生物医药 工程 ${ }^{[2]}$ 、药物运载 ${ }^{[3]}$ 、环境工程 ${ }^{[4]}$ 等各个方面具有良 好的应用前景。磁性纳米粒子的排布不同, 其对外 部磁场的响应也不相同, 磁性粒子的排布方式直接 决定着磁性水凝胶的性能。磁性粒子呈链状分布的 聚丙烯酰胺磁性水凝胶比磁性粒子无规则分布的磁 性水凝胶的磁热效应更为明显, 升温速率和平衡温 度分别提高了 8 倍和 2 倍 ${ }^{[5]}$ 。磁性粒子沿厚度方向 呈梯度分布的海藻酸钠磁性水凝胶, 相比于磁性粒子 无规则排布的磁性水凝胶具备更大的孔隙率, 在同样 的磁场强度下可产生更大的体积变形, 释放更多的水 分 $^{[6]}$ 。具有核壳结构的磁性粒子, 无磁场时, 磁性粒 子在磁性水凝胶中随机分布, 不会发生团聚; 施加磁 场后, 磁性粒子沿磁场方向分布, 形成周期结构。通 过外界激励改变核壳磁性粒子的排布间距, 进而引起 磁性水凝胶的颜色变化, 基于此制造了湿度传感器 ${ }^{[7]}$ 。 这种具有核壳结构的磁性粒子得到了广泛的研究, 被 用于制备多种光子晶体, 已被用于制作温度、压力、 湿度、化学传感器等 ${ }^{[7-8]}$ 。由此可见, 磁性粒子的排 布方式深刻影响着磁性水凝胶的功能。

目前, 对于其中磁性粒子分布的检出多用电镜 观测, 针对磁性水凝胶中磁性粒子检出的无损检测手 段基本空白, 基于磁性水凝胶在磁场下的产热特性, 可考虑用红外热成像法对其进行检测, 红外方法在金 属检测方面具有诸多应用，且取得了良好的结果 ${ }^{[9-11]}$ 。 因此, 本研究开发了基于交变磁场红外方法对磁性水 凝胶进行无损评价。本论文内容包括以下内容: 首先, 通过对磁性水凝胶和铜粉硅胶板的检测, 验证了磁性 水凝胶在交变磁场作用下的发热原理; 其次, 通过对 不同浓度磁性水凝胶的检测, 验证了利用交变磁场的 红外热成像对磁性水凝胶中磁性纳米粒子均匀性分 析的可行性, 并对磁性水凝胶中磁性粒子分布不均、 气泡缺陷分别进行了检测, 结果显示均能较好地检 出; 最后, 基于理论分析得出了磁性水凝胶在交变磁 场作用下的表面温升速率与其磁性纳米粒子浓度存 在线性关系的猜想, 通过对不同浓度水凝胶的检测得 到的数据进行拟合, 证实了磁性水凝胶中磁性纳米粒 子的浓度与其表面温升速率存在线性关系。

\section{1 方法论述}

\section{1 磁性水凝胶发热原理}

磁性粒子在交变磁场作用下, 由于磁滞效应和 弛豫效应, 会产生大量的热能。当磁性粒子处于单畴 尺寸时, 剩磁为 0 , 磁性粒子进入超顺磁状态。磁性 水凝胶中的磁性粒子为超细磁性纳米粒子, 在交变磁 场作用下没有磁滞效应, 只会发生弛豫耗散现象。

弛豫耗散产热的机理主要有两个方面, 一是磁 性粒子的布朗弛豫, 即磁性粒子由于黏性流动, 热 量直接传给流体; 二是磁性粒子的尼尔耗散, 即磁 性粒子在交变磁场作用下发生磁极的翻转, 产生热。 磁性水凝胶中磁性纳米粒子被自身高分子约束, 因 此后者是其发热原理，单位质量的磁性粒子的产热 量由式(1)得到 ${ }^{[12]}$

$$
P_{\text {Neel }}=\frac{\left(m H \omega \tau_{N}\right)^{2}}{2 \tau_{N} k T \rho V\left(1+\omega \tau_{N}{ }^{2}\right)^{2}}
$$

式中, $m$ 表示磁性粒子的磁矩; $H$ 表示磁场强度; $\omega$ 表示激励信号的角频率; $k$ 表示玻尔兹曼常数; $T$ 表示环境温度; $\rho$ 表示磁性粒子的密度; $V$ 表示磁性 粒子的体积; $\tau_{N}$ 表示尼尔耗散的弛豫时间, 由式(2) 求得 ${ }^{[12]}$

$$
\tau_{N}=\tau_{0} \exp \left(\frac{K V}{k T}\right)
$$

式中, $\tau_{0}$ 是一个时间常数, 取 $10^{-9} \mathrm{~s} ; K$ 表示各向 异性常数; $V$ 表示磁性粒子的体积; $k$ 表示玻尔兹曼 常数; $T$ 表示环境温度。

弛豫耗散会产生热量, 磁性粒子温度升高, 通 过热传导, 会使磁性粒子附近区域的磁性水凝胶基 质温度升高。磁性粒子分布密度较大的区域, 弛豫 耗散产生的热量相对较多, 使该区域温度升高相对 明显; 反之, 磁性粒子分布密度较小的区域, 温度 升高相对较小; 因此, 能够通过测量磁性水凝胶的 加热或冷却阶段的表面温度分布, 了解水凝胶中磁 性粒子的分布状况。

\section{2 试验平台搭建}

本文采用基于交变磁场的脉冲红外方法对磁性 水凝胶进行无损评价。该方法的试验装置由加热装 置、冷却装置、激励线圈、红外相机和数据采集系 统组成，如图 1 所示。实现该方法的过程如下：首 先将激励线圈放置在磁性水凝胶上方，利用冷却装 


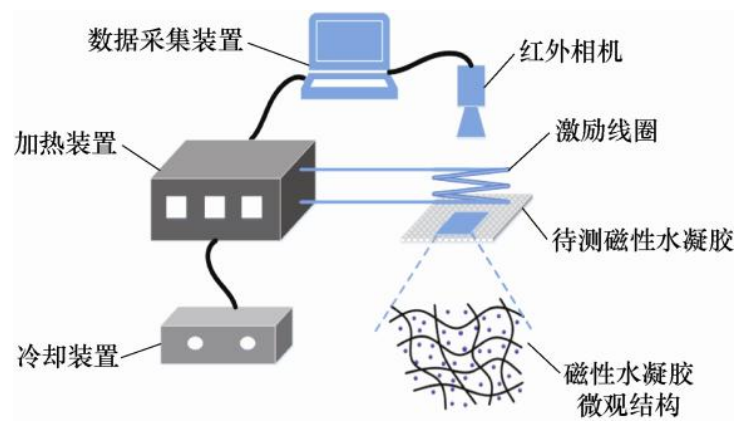

图 1 试验系统框架图

置对与加热装置相连的激励线圈进行冷却; 红外相 机放在磁性水凝胶上方, 与激励线圈保持一定的距 离; 然后利用加热装置给激励线圈施加交变激励电 流, 激励线圈会在空间中激发出一个变化的磁场, 激励线圈下的磁性水凝胶在磁场的作用下由于尼尔 弛豫耗散会产生热量; 与此同时, 给红外相机一个 采集信息的触发信号, 使红外相机的图像采集与施 加于激励线圈的激励信号实现同步, 触发信号采用 脉冲波, 可以控制红外相机采集图像的频率; 利用 数据采集装置记录红外相机采集到的图像序列; 最 后通过分析采集到的图像序列即可对磁性水凝胶中 磁性粒子的均匀性进行无损评价。

\section{2 试验结果}

\section{1 磁性水凝胶发热原理试验验证}

为了考察磁性水凝胶在交变磁场下的发热原 理, 本研究分别对磁性水凝胶和铜粉硅胶进行了检 测。磁性水凝胶中的磁性纳米粒子为 $\mathrm{Fe}_{3} \mathrm{O}_{4}$, 具有 磁性, 大尺度下具有良好的导电性能。铜粉硅胶中 的粒子为铜粉, 不具有磁性, 大尺度下也具有良好 的导电性能。磁性水凝胶中的磁性粒子和铜粉硅胶 中的铜粉, 尺寸均在 $10 \mathrm{~nm}$ 以下, 在纳米尺度下, 可以很好地比较不同粒子对磁场的响应情况。

对应两种不同的被测样品, 本研究设计了相对 于激励线圈不同的放置方式。本研究制备的磁性水 凝胶本身是没有黏性的, 并且由于磁性水凝胶极 易变形, 不能采用夹具, 因此, 考虑在线圈上方 平铺了一层保鲜膜, 将磁性水凝胶至于保鲜膜上, 如图 2 所示。保鲜膜本身对磁场没有响应, 且单 层薄膜不易传热, 因此对红外相机采集信息没有 干扰, 是很好的选择。铜粉硅胶本身是具有粘性的, 因此将其粘在一块挤压亚克力板上, 用夹具将亚克 力板支起来, 如图 3 所示。挤压亚克力板对磁场没 有反应, 且传热性能不佳, 夹具采用木制夹具, 对 磁场也没有响应。

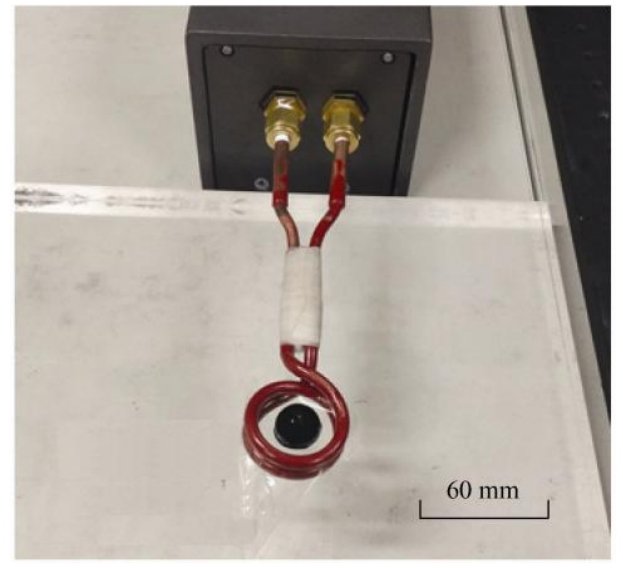

图 2 磁性水凝胶检测

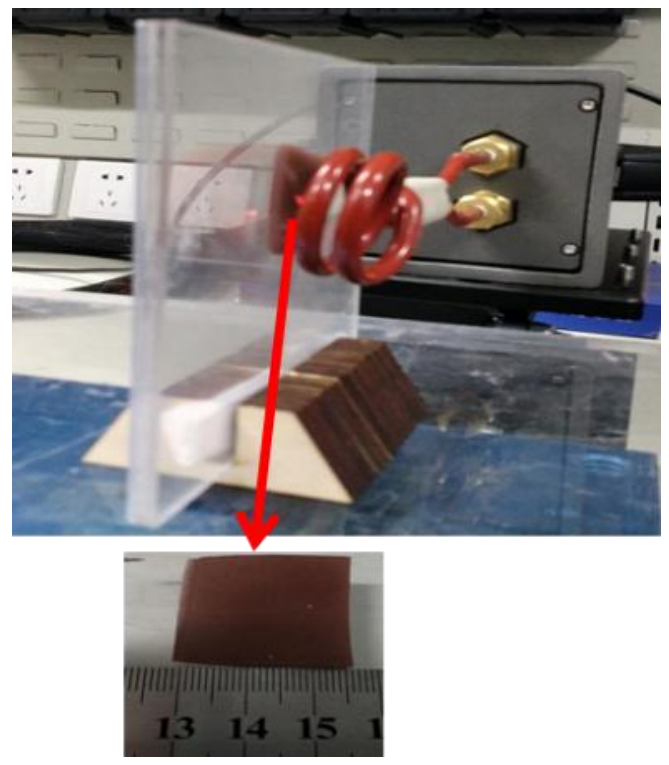

图 3 铜粉硅胶检测

图 4 和图 5 分别是磁性水凝胶和铜粉硅胶在试 验过程中的温度变化曲线, 在交变磁场下对两种材 料加热 $15 \mathrm{~s}$, 用红外相机采集数据 $30 \mathrm{~s}$, 可以看到, 磁性水凝胶对磁场响应明显, 温度明显升高, 超过 $10{ }^{\circ} \mathrm{C}$, 并且根据温度变化曲线可以看出, 有明显的

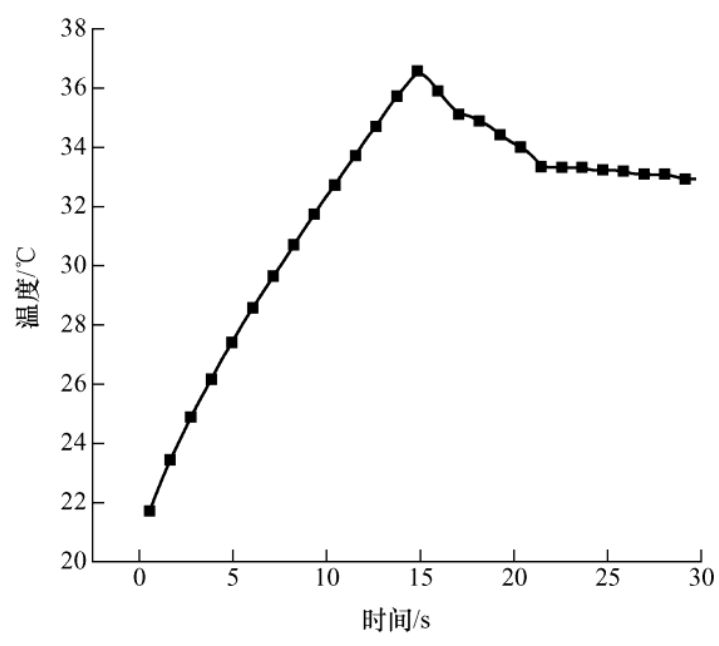

图 4 磁性水凝胶温度变化曲线 


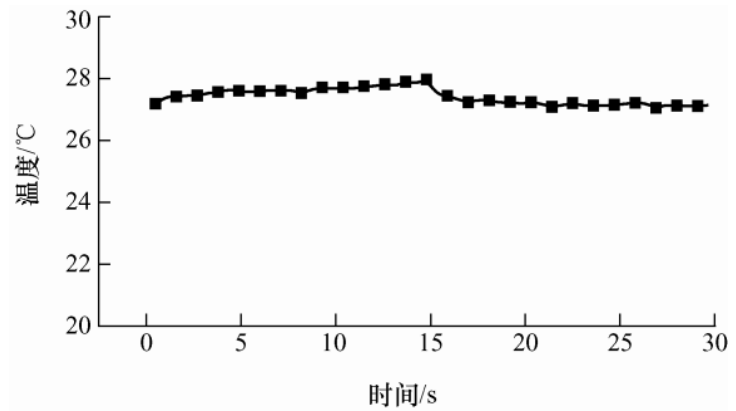

图 5 硅胶表面温度变化曲线

加热和散热过程。而铜粉硅胶对磁场基本没有响应, 整个加热过程温度基本不变, 由温度变化曲线也无 法观察到明显的加热和散热过程。

由图 4、5 所示的试验结果可知, 磁性水凝胶 中的磁性纳米粒子在交变磁场作用下有明显的发 热反应, 而铜粉硅胶中的铜粉粒子对交变磁场基 本没有响应, 说明纳米粒子材料的导电性对加热 过程没有影响, 对磁场响应起决定性作用的是纳 米粒子的磁性。

\section{2 磁性粒子含量不同检测试验}

磁性水凝胶一般由不同的单体以及磁性纳米 粒子(Magnetic nanoparticles, MNPs)组成, 而交联 剂的不同也会影响磁性水凝胶的性能。磁性水凝 胶常见的制备方法主要有三种 ${ }^{[13]}$, 分别是直接混 合法、原位沉淀法和嫁接法。直接混合法是将水 凝胶前体溶液与磁性纳米粒子直接混合得到磁性 水凝胶; 原位沉淀法是在水凝胶中导入金属离子, 然后通过碱处理得到磁性纳米粒子; 嫁接法是通 过官能团将磁性粒子与交联剂相连, 然后得到磁 性水凝胶。其中, 原位沉淀法在制备磁性水凝胶 方面具有几个优点。首先, 可以将大量无机颗粒 引入水凝胶网络中, 确保胶体中的颗粒可以很好 地分散在基质中, 得到的磁性水凝胶中的磁性纳 米粒子分布均匀; 其次, 制备的过程十分简单, 所需成本较低。因此, 本研究选择了一种利用原 位沉淀法制备的磁性水凝胶进行试验。在原位沉 淀法中, 首先, 通过自由基聚合制备水凝胶。然 后, 将水凝胶置于含有 $\mathrm{Fe}^{2+}$ 和 $\mathrm{Fe}^{3+}$ 的浓缩水溶液 中, 并以 $1: 2$ 的摩尔比取出亚铁离子直至达到溶 胀平衡。最后, 将吸收了 $\mathrm{Fe}^{2+}$ 和 $\mathrm{Fe}^{3+}$ 的溶胀水凝 胶浸入碱溶液中进行 MNP 沉淀。根据以下反应将 MNPs 制成水凝胶网络

$$
\mathrm{Fe}^{2+}+\mathrm{Fe}^{3+}+8 \mathrm{OH}^{-} \rightarrow \mathrm{Fe}_{3} \mathrm{O}_{4}(\downarrow)+4 \mathrm{H}_{2} \mathrm{O}
$$

该种磁性水凝胶中的磁性纳米粒子分布均匀, 磁性纳米粒子尺径为 $4.25 \mathrm{~nm} \pm 0.6 \mathrm{~nm}^{[14]}$ 。
将通过自由基聚合的水凝胶放入亚铁离子浓度 分别为 $0.075 \mathrm{~mol} / \mathrm{L}, 0.150 \mathrm{~mol} / \mathrm{L}, 0.225 \mathrm{~mol} / \mathrm{L}$, $0.300 \mathrm{~mol} / \mathrm{L}$ 的四种溶液中进行溶胀, 经过碱处理后 得到了四种磁性纳米粒子含量不同的磁性水凝胶。 本研究利用试验系统对四种 MNPs 含量不同的磁性 水凝胶进行了检测。图 6 是四种 MNPs 含量不同 的磁性水凝胶的温升曲线。可以看到, 在加热的 $15 \mathrm{~s}$ 内, MNPs 含量越高, 磁性水凝胶表面温度 越高, 且都有明显的加热过程, 散热过程由于温 度差的影响, 可以看到, 温升越多的磁性水凝胶, 散热效果越明显。为了考察磁性水凝胶温升与磁 性纳米粒子含量的关系, 本研究对发热过程进行 分析。由式(2)可知, 弛豫时间均与环境温度 $T$ 有 关。由于磁性纳米粒子半径很小, 因此, 弛豫时 间都很短, 耗散过程可以看成准静态过程。对式(1) 进行分析可以得到。 $p \propto 1 / T$ 如果 $T$ 的变化不大, 整个加热过程中, 磁性纳米粒子的耗散功率可以 看成是恒定的。事实上, 在本研究的试验过程中, 整体加热时间较短, 样品温度变化不超过 $20{ }^{\circ} \mathrm{C}$, 因此, 整个过程中，每个磁性纳米粒子的发热量 可以看作是不变的。

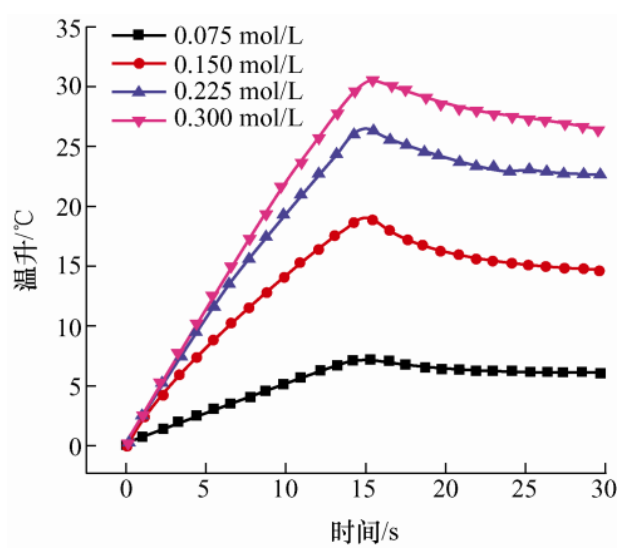

图 6 不同含量水凝胶温升曲线

同时本研究考虑样品本身的散热效应。在整个 加热过程中, 随着样品温度的升高, 与环境温差会 越来越大, 由于温度差的增加, 样品的散热就会越 快, 因此, 样品自身的温升速率肯定会下降。为了 消除散热的影响, 本研究可以采用温升曲线开始段 的斜率作为评价量, 即斜率越大, 磁性纳米粒子的 浓度越高。根据分析, 粒子浓度与样品温升速率的 关系应该符合式(4)

$$
C_{\text {particle }}=F \cdot V_{\text {temp }}
$$

式中, $C_{\text {particle }}$ 表示磁性水凝胶中磁性粒子的含量, $V_{\text {temp }}$ 表示交变磁场作用下磁性水凝胶表面温升速 
率, $F$ 表示磁性粒子含量-磁性水凝胶表面温升速率 模型系数。图 7 是对 MNPs 含量与温升速率的拟合 结果, 在拟合过程中, 将无磁性粒子的水凝胶加入, 即加入 $(0,0)$ 点进行拟合, 可以看到 MNPs 含量与 温升速率呈现线性关系, 与分析相符。因此, 通过 加热过程磁性水凝胶表面温升曲线的斜率来评价磁 性水凝胶中磁性纳米粒子的浓度是完全可行的。

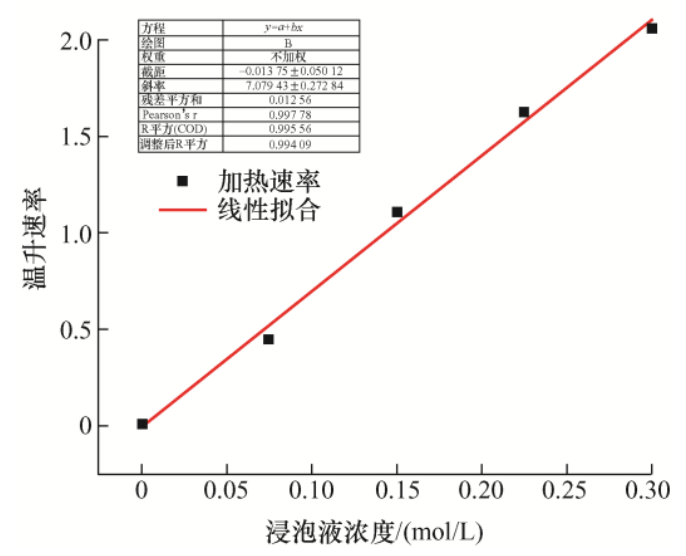

图 7 磁性粒子含量与温升速率曲线

\section{3 气泡及磁性粒子分布不均检测试验}

本研究针对磁性水凝胶中容易出现的气泡缺 陷，以及 MNPs 不同分布的情况进行了检测试验。 图 8 是针对气泡缺陷的检出结果。可以看到, 在加 热过程中, 有气泡处和无气泡处温升具有一定的差 异性, 无气泡处的温升明显比有气泡处快, 但是, 两者之间温差较小, 不超过 $1{ }^{\circ} \mathrm{C}$ 。而在散热过程中 可以明显的看到, 有气泡处温度高于无气泡处的温 度, 温差超过 $3{ }^{\circ} \mathrm{C}$ 。加热时, 气泡外的磁性水凝胶 中的磁性纳米粒子会对磁场做出响应, 使该部分温 度升高, 红外相机采集到的信息为表面温度信息, 虽然气泡影响了发热效率, 但是并不显著, 因此很

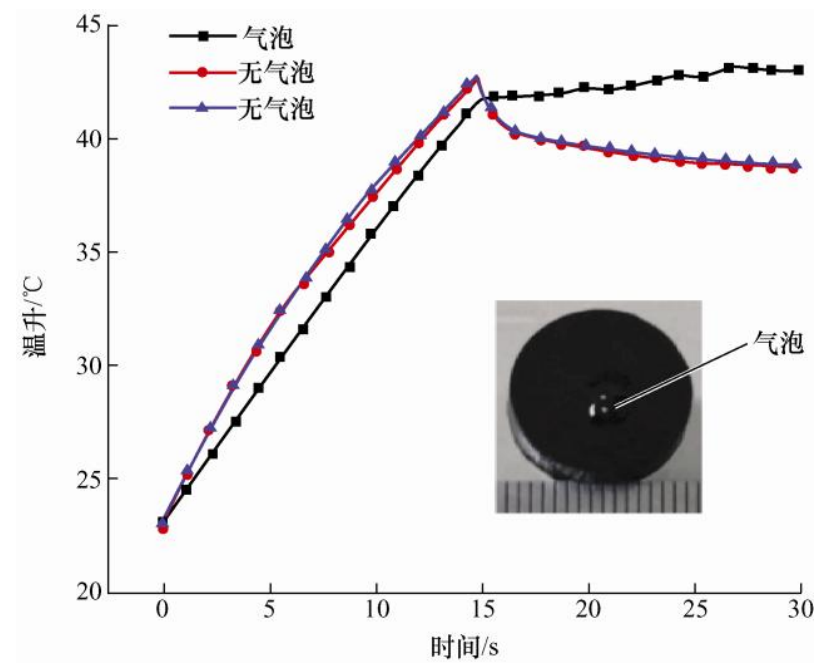

图 8 气泡缺陷的检测结果
难直接在加热过程中通过观察红外相机拍摄的照片 观测到气泡的存在。而当整片磁性水凝胶处于散热 过程时, 气泡空腔中的区域在加热过程中也被加热, 整体温度较高, 导致在整个散热过程中，气泡区域 呈现出保温状态, 温度没有明显的变化。因此, 综 合加热以及散热过程的信息，该种无损检测方法可 以对磁性水凝胶中的气泡缺陷进行有效的检出。

本研究针对 MNPs 的分布制备了不同的磁性水 凝胶, 图 9a 制备时将水凝胶表层全部浸入碱溶液生 成磁性纳米粒子, 图 9d 制备时将水凝胶表层圆环区 域浸入碱溶液生成磁性纳米粒子。将得到的样品进 行检测, 结果如图 9 所示。由于加热 $15 \mathrm{~s}$ 及 $60 \mathrm{~s}$ 时红外图像对比度更好, 更利于观察, 因此选取了 这两个时刻的红外图像。通过检测得到的红外图像 可以看到, 无论是表层全部浸入磁性粒子的水凝胶, 还是表面磁性粒子为环状区域的水凝胶, 均只在存 在磁性粒子的区域有明显的发热现象，且加热时间 越长, 磁性粒子发热越明显。图 9b、9c 可以看到水 凝胶表层发热较快, 在加热 $15 \mathrm{~s}$ 时, 无磁性粒子的 水凝胶区域基本不发热, 出现了明显的分层现象。 图 9e、9f 可以看到加热过程中出现了明显的圆环形 状, 且在加热 $15 \mathrm{~s}$ 时, 没有磁性粒子的水凝胶区域 没有发热现象。结合对两种分布的检测结果可以看 到, 无磁性粒子区域的水凝胶在 $60 \mathrm{~s}$ 时存在的温升 是由于传热导致的, 通过红外图像可以直接观测得到 磁性粒子的分布状况。因此，该无损检测方法对两种 磁性粒子分布不均的情况均可以较好地检出。

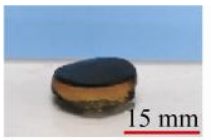

(a) 圆形样品

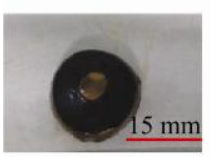

(d) 环形样品

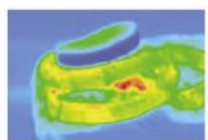

(b) 加热 $15 \mathrm{~s}$ 温度图

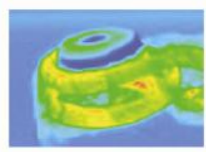

(e) 加热 $15 \mathrm{~s}$ 温度图

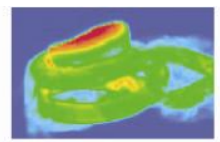

(c) 加热60 s 温度图

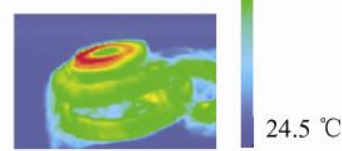

(f) 加热60 s温度图 (g) 温度条
图 9 MNPs 不同分布时的检测结果

\section{3 结论}

本文首先基于磁性水凝胶在交变磁场下的发热 原理搭建了交变磁场红外的无损检测试验系统; 其 次通过对不同磁性粒子含量的磁性水凝胶的检测确 定了对其磁性粒子含量检测的可行性, 并且提出了 利用磁性水凝胶在交变磁场下的发热速率来确定其 磁性粒子含量的方法; 最后基于对磁性水凝胶中气 
泡缺陷以及磁性粒子分布不均的检测结果, 验证了 所开发的交变磁场红外检测方法的有效性, 填补了 磁性水凝胶缺陷检测方法的空白。

\section{参 考 文 献}

[1] KIM Y S, LIU M, ISHIDA Y, et al. Thermoresponsive actuation enabled by permittivity switching in an electrostatically anisotropic hydrogel[J]. Nature Materials, 2015, 14(10): 1002-1007.

[2] KIM J I, CHUN C J, KIM B, et al. Thermosensitive/magnetic poly(organophosphazene) hydrogel as a long-term magnetic resonance contrast platform[J]. Biomaterials, 2012, 33(1): 218-224.

[3] SATARKAR N S, HILT J Z. Magnetic hydrogel nanocomposites for remote controlled pulsatile drug release[J]. Journal of Controlled Release, 2008, 130(3): 246-251.

[4] 华蓉. 磁性水凝胶的制备及其对重金属的吸附研究 [D]. 南京: 南京大学, 2014.

HUA Rong. Synthesis and heavy metal ion adsorption studies of a magnetic hydrogel[D]. Nanjing: Nanjing University, 2014.

[5] HU Ke, SUN Jianfei, GUO Zhaobin, et al. A novel magnetic hydrogel with aligned magnetic colloidal assemblies showing controllable enhancement of magnetothermal effect in the presence of alternating magnetic field[J]. Advanced Materials, 2015, 27: 2507-2514.

[6] CEZAR C, KENNEDY SM, MEHTA M, et al. Biphasic ferrogels for triggered drug and cell delivery[J]. Advanced Healthcare Materials, 2014, 3(11): 1869-1876.

[7] HU Haibo, CHEN Qianwang, CHENG Kai, et al. Visually readable and highly stable self-display photonic humidity sensor[J]. Journal of Materials Chemistry, 2012, 22: 1021-1027.

[8] GE Jianping, YIN Yadong. Responsive photonic crystals[J]. Angewandte Chemie International Edition, 2011,
50(7): 1492-1522.

[9] 郭兴旺, 李涁. 重型铝合金结构裂纹振动红外热像检测 的建模和分析 [J]. 机械工程学报, 2014, 50(24): 31-37. GUO Xingwang, LI Bin. Modeling and analysis of vibrothermography of cracks in heavy aluminum alloy structures[J]. Journal of Mechanical Engineering, 2014, 50(24): 31-37.

[10] 张超省, 冯辅周, 闵庆旭, 等. 超声红外热像技术中预 紧力对金属平板振动特性的影响 $[\mathrm{J}]$. 机械工程学报, 2016, 52(5): 154-161.

ZHANG Chaosheng, FENG Fuzhou, MIN Qingxu, et al. Effect of engagement force on vibration characteristics of metal plate in sonic infrared imaging[J]. Journal of Mechanical Engineering, 2016，52(5): 154-161.

[11] 陆向宁, 何贞志, 胡宁宁, 等. 主动红外热成像焊球缺 陷检测方法研究[J]. 机械工程学报, 2016, 52(10): 17-24. LU Xiangning, HE Zhenzhi, HU Ningning, et al. Research on defects inspection of solder bumps using active infrared thermography technology[J]. Journal of Mechanical Engineering, 2016, 52(10): 17-24.

[12] HERGT R, ANDRA W, D AMBLY C G, et al. Physical limits of hyperthermia using magnetite fine particles[J]. IEEE Transactions on Magnetics, 1998, 34(5):3745-3754.

[13] LI Yuhui, HUANG Guoyou, ZHANG Xiaohui, et al. Magnetic hydrogels and their potential biomedical applications[J]. Advanced Functional Materials, 2013, 23(6): 660-672.

[14] TANG Jingda, TONG Zongfei, XIA Yukun, et al. Super tough magnetic hydrogels for remotely triggered shape morphing[J]. Journal of Materials Chemistry B, 2018, 6(18): 2713-2722.

作者简介: 乔严程, 男, 1996 年出生。主要研究方向为软物质与红外无 损检测方法。

E-mail: qiaoyancheng123@stu.xjtu.edu.cn

解社娟(通信作者), 女, 1983 年出生, 博士, 副教授, 博士研究生导师。 主要研究方向为基于电磁/声/热的无损检测与断裂力学。

E-mail: xiesj2014@mail.xjtu.edu.cn 